\title{
Electrostatic Regulation of Quality Factor in Non-Ideal Tuning Fork MEMS
}

\author{
Alexander. A. Trusov, Sergei A. Zotov, Andrei M. Shkel \\ MicroSystems Laboratory, Mechanical and Aerospace Engineering \\ University of California, Irvine, CA, USA \\ atrusov@uci.edu,szotov@uci.edu, ashkel@uci.edu
}

\begin{abstract}
This paper presents a method of regulation and maximization of Q-factor in anti-phase driven tuning fork MEMS. Non-symmetric regulation of stiffness in coupled 2-DOF resonators using the negative electrostatic spring effect is shown to adjust the momentum misbalance caused by fabrication imperfections in nominally symmetric structures. Balancing the structure through stiffness matching minimizes the loss of energy through the substrate and maximizes the device antiphase Q-factor. The approach is experimentally demonstrated using a vacuum packaged SOI MEMS tuning fork gyroscope with initial operational frequency of $2.2 \mathrm{kHz}$ and Q-factor of 0.6 million. By electrostatically tuning stiffness of one of the suspensions, momentum misbalanced caused by the fabrication imperfections was minimized, suppressing anchor loss and increasing the Q-factor to above 0.8 million, attributed to the thermoelastic limit. The experimentally validated analytical model of substrate dissipation is applicable to Q-factor tuning in anti-phase resonators and gyroscopes.
\end{abstract}

\section{INTRODUCTION}

Maximization of the mechanical quality factor is critical for improving the performance of micromachined vibratory transducers [1]. Resonant accelerometers, vibratory gyroscopes, RF filters, and resonant chemical sensors all benefit from high Q-factor architectures [2]. Energy dissipation in vibratory MEMS is governed by several mechanisms, including viscous damping, dissipation through substrate (anchor loss), Thermo-Elastic Dissipation (TED), electronic losses, and surface effects [3]. When air damping is minimized through vacuum packaging, the device Q-factor is typically limited by the substrate dissipation and TED.

One common approach for reduction of the substrate energy dissipation is based on balanced tuning fork architectures $[3,4]$, where two coupled masses (tines) are driven in anti-phase motion to minimize the net momentum applied to the substrate [5]. At the same time, an ideally balanced tuning fork provides rejection of common mode external accelerations. Imperfections are commonplace in MEMS fabrication technologies and often prevent exact realization of the nominal mass and stiffness values, causing structural non-symmetries in fabricated devices. As a result, the frequency response characteristics of each tine are slightly different and a non-zero net momentum is created causing the dissipation of energy through the substrate [5] as well as an undesired susceptibility to common mode acceleration inputs.

This work was supported by the ONR/NSWCDD under grants N0001409-1-0424 and N00014-11-1-0483.



Figure 1. Concept of anti-phase Q-factor tuning: matching tine stiffness eliminates anchor loss and maximizes Q-factor to the thermoelastic limit.

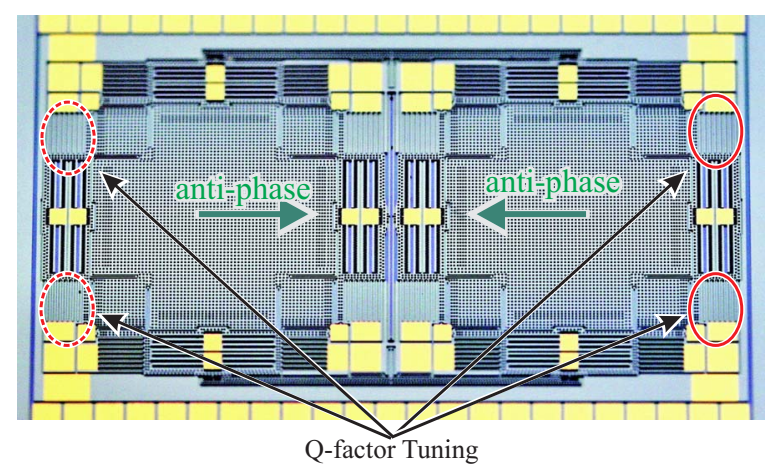

Figure 2. Optical photograph of a dual mass anti-phase gyroscope used for the experimental study of substrate dissipation and Q-factor tuning.

The anti-phase resonance of a non-ideal tuning fork can be analyzed as a superposition of an ideally balanced anti-phase and an in-phase motion. The parasitic in-phase mode accounts for the structural misbalance and allows the substrate energy dissipation. This energy loss can be minimized by matching the suspension stiffnesses of the two tines post-fabrication via the voltage-controlled negative electrostatic spring effect. At the same time, balancing of a tuning fork structure improves the common mode rejection.

This paper provides an analytical and experimental analysis of the effect of structural imbalance in anti-phase tuning forks on the dissipation of energy through the substrate. A theoretical model for the anchor loss Q-factor as a function of the stiffness mismatch is developed, Fig. 1. A method of Qfactor regulation based on stiffness matching through the negative electrostatic spring effect is proposed and validated. 


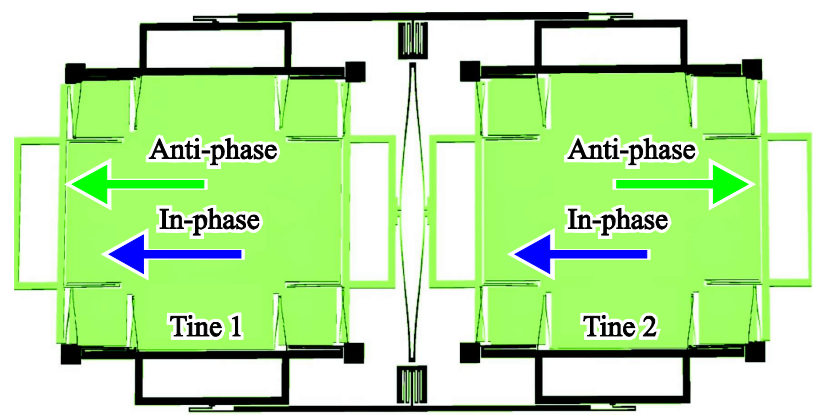

Figure 3. FEM modeling of the dual mass tuning fork gyroscope sensemode, illustrating the main anti-phase and undesired in-phase motion.

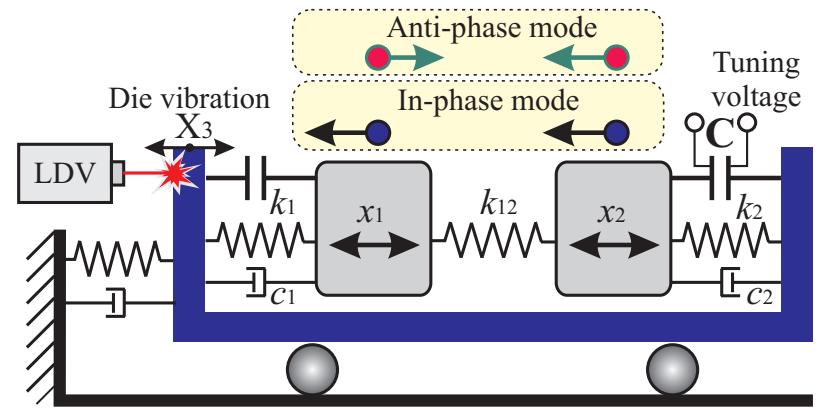

Figure 4. Schematic of the Q-factor tuning in an anti-phase dual mass device based on selective electrostatic tuning and matching of stiffnesses.

\section{EXPERIMENTAL TESTBED}

In this section we describe the gyroscope used for the study of substrate dissipation and Q-factor regulation.

\section{A. Transducer Design}

A low dissipation dual mass tuning fork SOI gyroscope [6] was used for the experimental study of substrate dissipation and Q-factor regulation via electrostatic tuning, Fig. 2. The gyroscope is comprised of two symmetrically decoupled tines, and coupling flexures for the linear anti-phase sense-mode Fig. 2. Each tine is an $x-y$ isotropic resonator consisting of a square proof mass suspended by two drive-mode and two sense-mode decoupling shuttles. Each tine has a number of parallel plate electrodes allowing tuning of individual stiffnesses through the negative electrostatic spring effect.

The sense-mode of the gyroscope, used for the Q-factor study, is formed by the two linearly coupled tines moving along a line in anti-phase to each other, Fig. 3. Unlike conventional tuning fork gyroscopes, the proposed architecture prioritizes the quality factor of the sense-mode by mechanical design, where the linearly coupled anti-phase sense-mode is nominally balanced in both the linear momentum as well as torque in order to minimize the dissipation of energy through the substrate.

\section{B. Transducer Fabrication}

The tuning fork gyroscopes used for the experimental characterization of Q-factors were fabricated in-house using a single mask SOI process with a conductive, $100 \mu \mathrm{m}$ thick device layer and a $5 \mu \mathrm{m}$ thick buried oxide layer. After a $1 \mu \mathrm{m}$ surface oxide layer is thermally grown on the device layer, this

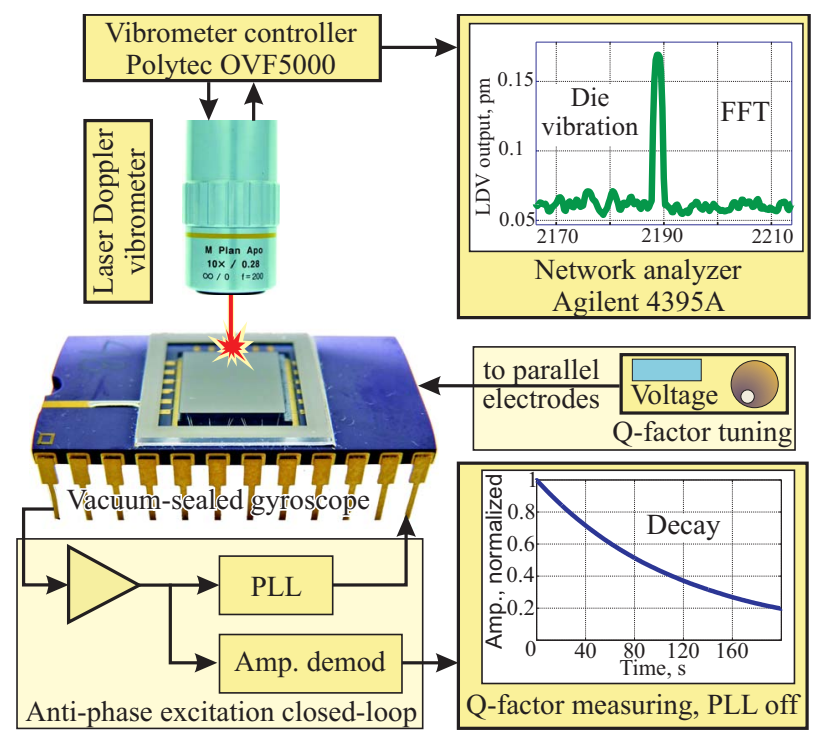

Figure 5. Schematic of the Q-factor tuning characterization experiment using a vacuum packaged dual mass gyroscope and a laser vibrometer.

layer is patterned and etched through the use of a Surface Technology Systems Advanced Oxide Etching tool to define a hard mask prior to the etching of the device layer. Following dicing, singular sensors were released using a timed $20 \%$ hydrofluoric acid wet etch. The released devices were bonded to ceramic DIP packages using Au-Sn eutectic solder and wire bonded. Sensors were vacuum sealed with getters under submTorr pressure for standalone high Q-factor operation [7].

\section{EXPERIMENTAL CHARACTERIZATION}

In this section we report the experimental testing of the stiffness tuning effect on the substrate energy dissipation.

\section{A. Substrate Energy Dissipation and Q-factor Tuning}

The sense-mode of a vacuum packaged SOI tuning fork gyroscope was used for the experimental characterization of the substrate dissipation and Q-factor maximization. The sense-mode resonator was excited into the anti-phase vibrations using a combination of a constant $\mathrm{DC}$ polarization voltage and an AC voltage generated by a PLL. A carrier of 1 Vrms at $52 \mathrm{kHz}$ was applied to the proof mass, resulting in the amplitude modulation of the signal corresponding to the velocity of the proof mass. The motional signal from detection capacitors was amplified and amplitude demodulated twice (first at the carrier frequency and then at the anti-phase resonance frequency) in order to extract the amplitude of the anti-phase motion. A Polytec OVF-5000 single-point Laser Doppler Vibrometer pointed at the edge of the die through an optical window on the vacuum package was used to directly measure the vibration of the substrate, Fig. 4.

The following procedure was used to determine the vibrational amplitude of die substrate and device Q-factor for various values of a voltage-controlled negative electrostatic spring, Fig. 5.

1) Apply a DC voltage to the stiffness tuning parallel plate electrodes on one of the tines. 


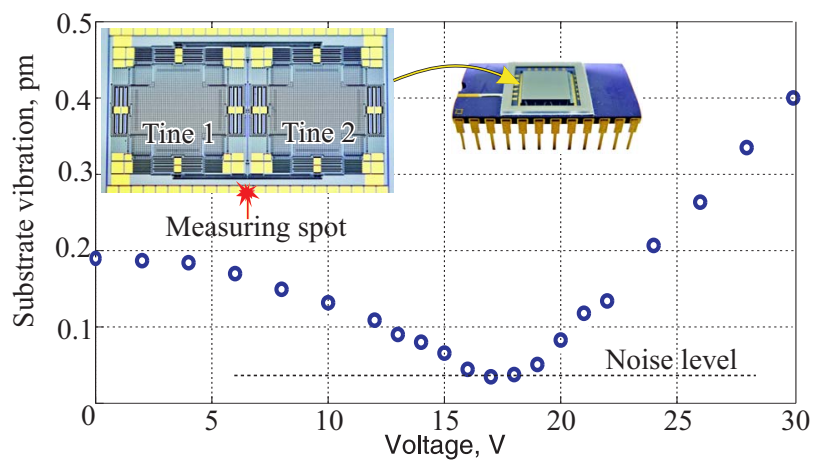

(a) Measured substrate vibration for different tuning voltage. Stiffness matching minimizes transfer of energy to the substrate (anchor loss).

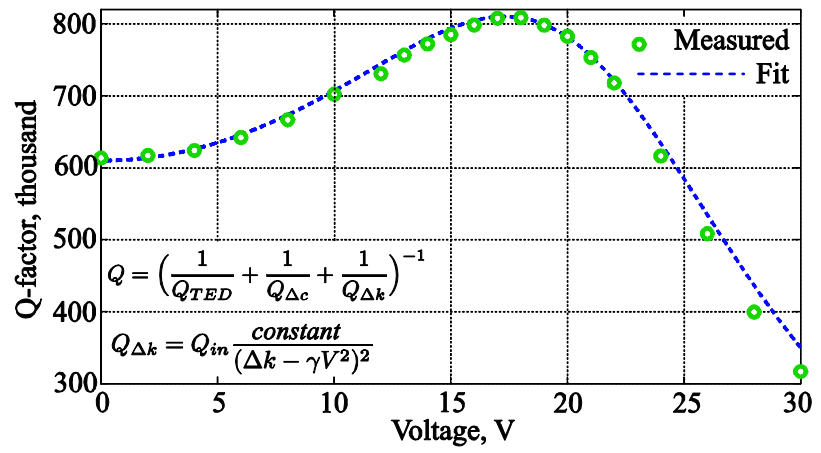

(b) Measured Q-factor of the anti-phase mode. Tine stiffness matching maximizes Q-factor to the TED limit by minimizing substrate loss.

Figure 6. Characterization of the stiffness matching effect on substrate vibration and gyroscope Q-factor (tine matching is achieved at $18 \mathrm{~V}$ ).

2) Excite the anti-phase mode of the gyroscope into resonance with an amplitude of several microns.

3) Measure vibrational amplitude of the substrate die using the laser vibrometer.

4) Turn off the excitation voltage, record free vibrations time history, and extract the Q-factor from the decay.

5) Change the value of the stiffness tuning DC voltage and repeat steps $1-4$.

Measurements of the amplitude of the substrate vibrations and the tuning fork anti-phase Q-factors for different stiffness misbalances are shown in Fig. 6. Initially, the sense-mode of the gyroscope has an anti-phase Q-factor of 0.6 million limited by anchor loss due to fabrication imperfections. At the same time, considerable substrate vibration is detected by the vibrometer. Increasing the tuning voltage on the stiffer tine reduces the misbalance, resulting in substrate vibrations reduction and Q-factor increase. At approximately $18 \mathrm{~V}$ the two tines are matched, producing minimal substrate vibration (on the level of noise). At the same time, the Q-factor is increased to 0.8 million, which agrees well with the fundamental thermoelastic limit computed using FEM software COMSOL. Increasing the stiffness tuning voltage above $18 \mathrm{~V}$ perturbs the symmetry, introducing energy dissipation through the substrate back into the dual system.

The data illustrates that minimization of anchor loss requires structural balancing of the tuning fork structure and

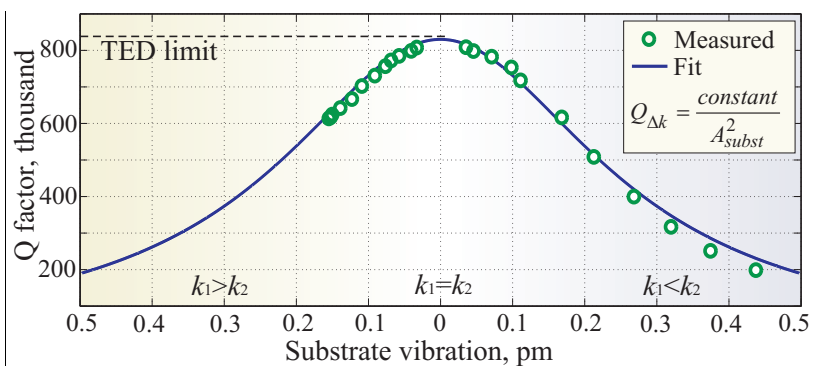

Figure 7. Measured anti-phase Q-factor versus substrate vibration, illustrating the effect of structural misbalance on substrate dissipation.

suppression of the die substrate vibrations, Fig. 7. The negative electrostatic spring effect allows for post-fabrication voltage-controlled balancing of tuning fork resonators.

\section{TheOretical Model For SUbSTRATE Dissipation}

In this section we present a mathematical model for the substrate dissipation. The model quantitatively explains the effect of structural balancing on Q-factor maximization.

\section{A. Equations of Motion}

Fig. 4 shows a conceptual schematic used to analytically describe the effects of the stiffness tuning process on the dissipation of energy through the substrate. Positions of the two tines are described by coordinates $x_{1}$ and $x_{2}$, respectively. Assuming the tines are initially matched in mass, the positions of the center of mass of the two tines is given by $y_{i n}=\left(x_{1}-x_{2}\right) / 2$, which represents the amount of the undesired in-phase motion. The amount of the primary anti-phase motion is given by $y_{a n}=\left(x_{1}+x_{2}\right) / 2$. The equations of motion of the tuning fork in the generalized coordinates $y_{i n}$ and $y_{a n}$ can be derived as

$$
\begin{aligned}
& \ddot{y}_{a n}+\frac{\omega_{a n}}{Q_{a n}} \dot{y}_{a n}+\omega_{a n}^{2} y_{a n}=\frac{\Delta c \dot{y}_{i n}+\Delta k y_{i n}}{2 m}+\frac{F(t)}{2 m}, \\
& \ddot{y}_{i n}+\frac{\omega_{i n}}{Q_{i n}} \dot{y}_{i n}+\omega_{i n}^{2} y_{i n}=\frac{\Delta c \dot{y}_{a n}+\Delta k y_{a n}}{2 m},
\end{aligned}
$$

where $m$ is the mass of each tine, $\omega_{i n}, \omega_{a n}$ and $Q_{i n}, Q_{a n}$ are the decoupled natural frequencies and Q-factors of the ideal antiphase and in-phase motion, respectively; $F(t)$ is the anti-phase excitation force; $\Delta c$ and $\Delta k$ are the damping and stiffness mismatch, respectively. In case of nominally matched tuning forks with slight misbalances, it can be assumed that $y_{a n}>>y_{i n}$, which allows neglecting the term $\left(\Delta c y^{\prime}{ }_{i n}+\Delta k y_{i n}\right) / 2 m$ in (1).

\section{B. Solution of the Equations of Motion}

The tuning fork resonator is designed to be driven in the anti-phase resonance. We assume that the anti-phase component $y_{a n}$ is given by a sinusoid with amplitude $A_{a n}$ and frequency $\omega_{a n}$ :

$$
y_{a n}=A_{a n} \sin \left(\omega_{a n} t\right) .
$$

Substituting (2) into (1), solution for the in-phase component of motion $y_{i n}$ is

$$
y_{i n}=A_{a n} \frac{\Delta k \sin \left(\omega_{a n} t+\phi\right)+\Delta c \omega_{a n} \cos \left(\omega_{a n} t+\phi\right)}{2 m \sqrt{\left(\omega_{i n}^{2}-\omega_{a n}^{2}\right)^{2}+\omega_{i n}^{2} \omega_{a n}^{2} / Q_{i n}^{2}}}
$$

As (3) shows, the contributions of the damping and stiffness mismatches, $\Delta c$ and $\Delta k$, are linearly independent and the superposition principle can be used. Further analysis will 
focus separately on the effects of damping and stiffness mismatch on the substrate dissipation.

\section{Effect of Anchor Loss on the Anti-Phase Q-Factor}

To analyze the dissipation of energy through the substrate due to misbalances in a tuning fork resonator we solve (1)-(3) for the amount of energy dissipated during one cycle of motion. The dissipation of energy per cycle of the anti-phase motion $\Delta E_{\text {an }}$ is given by the integral of the dissipative term $\omega_{\text {an }} y^{\prime}$ an $/ Q_{\text {an }}$ multiplied by the anti-phase velocity $y_{\text {an }}^{\prime}$ :

$$
\Delta E_{a n}=\int_{0}^{2 \pi / \omega_{a n}} \frac{\omega_{a n}}{Q_{a n}} \dot{y}_{a n}^{2}=A_{a n}^{2} \pi \frac{\omega_{a n}^{2}}{Q_{a n}},
$$

where it is assumed that $\Delta c<<c$. Similarly, the energy dissipated per cycle for the in-phase mode is calculated as:

$$
\begin{aligned}
& \Delta E_{i n}=\int_{0}^{2 \pi / \omega_{a n}} \frac{\omega_{i n}}{Q_{i n}} \dot{y}_{i n}^{2}= \\
& =\pi \frac{A_{a n}^{2}}{Q_{i n}} \frac{\left.\Delta k^{2} \omega_{i n} \omega_{a n}+\Delta c^{2} \omega_{i n} \omega_{a n}^{3}\left(\omega_{i n}^{2}-\omega_{a n}^{2}\right)^{2}+\omega_{i n}^{2} \omega_{a n}^{2} / Q_{i n}^{2}\right)}{4 m^{2}} .
\end{aligned}
$$

At the same time, the total mechanical energy in the system can be approximated by the energy stored by the antiphase mode of vibrations (assuming $y_{a n}>>y_{i n}$ ):

$$
E_{\text {Stored }}=\frac{1}{2}\left(A_{a n} \omega_{a n}\right)^{2} \text {. }
$$

Finally, the quality factor is defined as the ratio between stored and dissipated energy per one cycle of vibrations multiplied by $2 \pi$ :

$$
Q=2 \pi \frac{\text { Energy stored }}{\text { Energy dissipated per cycle }} .
$$

The anti-phase quality factor corresponding to the dissipation of energy through the substrate due to structural misbalances can now be found by substituting the energy expressions (4) and (6) into Q-factor definition (7). For the case of stiffness mismatch $\Delta k \neq 0$ (assuming zero damping mismatch $\Delta c$ ),

$$
Q_{\Delta k}=Q_{i n} \frac{4 m^{2}}{\Delta k^{2}} \frac{\omega_{a n}\left(\left(\omega_{i n}^{2}-\omega_{a n}^{2}\right)^{2}+\omega_{i n}^{2} \omega_{a n}^{2} / Q_{i n}^{2}\right)}{\omega_{i n}}
$$

Similarly, for the case of damping mismatch $\Delta c \neq 0$ (assuming zero stiffness mismatch $\Delta k$ )

$$
Q_{\Delta c}=Q_{i n} \frac{4 m^{2}}{\Delta c^{2}} \frac{\left(\left(\omega_{i n}^{2}-\omega_{a n}^{2}\right)^{2}+\omega_{i n}^{2} \omega_{a n}^{2} / Q_{i n}^{2}\right)}{\omega_{a n} \omega_{i n}} .
$$

The total Q-factor of the non-ideal coupled anti-phase motion is a function of the decoupled ideal anti-phase $Q_{a n}$ and anti-phase Q-factors corresponding to the anchor loss due to structural misbalances $\Delta k$ and $\Delta c$,

$$
Q_{\text {Total }}=\left(\frac{1}{Q_{a n}}+\frac{1}{Q_{\Delta k}}+\frac{1}{Q_{\Delta C}}\right)^{-1} .
$$

Expressions (8)-(10) constitute a mathematical model for energy dissipation in non-ideal tuning fork resonators. For practical purposes, the decoupled Q-factor, $Q_{a n}$, of the ideal anti-phase mode is calculated as the thermoelastic limit of the structure.

\section{Application of the Model to Experimental Data}

The derived analytical model for energy dissipation in non-ideal tuning forks allows quantitative analysis of the experimental data in Fig. 6 and Fig. 7. In these experiments, the effective stiffness mismatch $\Delta k$ becomes a function of the negative electrostatic spring tuning voltage. Fitting the data with the analytical model (8), (10) reveals a good agreement, validating the developed mathematical model. Fitting of the data also allows quantitative identification of the system misbalance (for the device under test, the relative stiffness mismatch $\Delta k /(k 1+k 2)$ of $0.75 \%$ was identified).

\section{CONCLUSION}

We investigated the dissipation of energy through the substrate in non-ideal anti-phase operated tuning fork MEMS. A mathematical model was developed for Q-factor of nonideal resonators as a function of stiffness and damping imbalance. Reduction of the structural imbalances for Q-factor maximization was proposed based on post-fabrication electrostatic tuning of stiffness. The model was validated using a vacuum packaged SOI MEMS tuning fork gyroscope with initial operational frequency of $2.2 \mathrm{kHz}$ and Q-factor of 0.6 million. By electrostatically tuning one of the suspension springs, momentum misbalanced caused by the fabrication imperfections was minimized, suppressing anchor loss and increasing the Q-factor above 0.8 million. The substrate dissipation model, validated by experiments, is valuable for trimming and tuning of Q-factors in anti-phase resonators and gyroscopes.

\section{ACKNOWLEDGMENT}

This work was funded by ONR/NSWCDD grants N0001409-1-0424 and N00014-11-1-0483. The authors would like to acknowledge Igor Prikhodko for assistance with the experimental characterization of the prototype and Ilya Chepurko for assistance with the interface electronics. The gyroscopes were designed and characterized at the MicroSystems Laboratory, University of California, Irvine.

\section{REFERENCES}

[1] A.A. Trusov, I.P. Prikhodko, S.A. Zotov, A.R. Schofield, and A.M. Shkel, "Ultra-high Q silicon gyroscopes with interchangeable rate and whole angle modes of operation," IEEE Sensors 2010 Conference, Waikoloa, Hawaii, USA, November, 2010.

[2] N. Yazdi, F. Ayazi, and K. Najafi, "Micromachined inertial sensors," Proc. IEEE, vol. 86, no. 8, pp. 1640-1659, Aug. 1998.

[3] M. Weinberg, R. Candler, S. Chandorkar, J. Varsanik, T. Kenny, and A. Duwel, "Energy loss in MEMS resonators and the impact on inertial and RF devices," Proc. Transducers 2009, pp. 688-695.

[4] M. Weinberg, A. Kourepenis, "Error sources in in-plane silicon tuningfork MEMS gyroscopes," JMEMS Vol. 15, No. 3, June 2006.

[5] A.A. Trusov, A.R. Schofield, A.M. Shkel, "A substrate energy dissipation mechanism in in-phase and anti-phase micromachined Zaxis vibratory gyroscopes," JMM, vol. 18, pp. 095016(10), September 2008.

[6] A.A. Trusov, A.R. Schofield, and A.M. Shkel, "Gyroscope Architecture with Structurally Forced Anti-Phase Drive-Mode and Linearly Coupled Anti-Phase Sense-Mode," Proc. Transducers 2009, pp. 660-664.

[7] A.R. Schofield, A.A. Trusov, and A.M. Shkel, "Versatile Sub-mTorr Vacuum Packaging for the Experimental Study of Resonant MEMS," Proc. IEEE MEMS 2010, pp. 516-519, 2010. 